\title{
Transmitter-Preprocessing-Assisted Cooperative Downlink Transmission in DS-CDMA Systems Experiencing Propagation Path Loss and Nakagami- $m$ Fading
}

\author{
Wei Fang, Associate Member, IEEE, Lie-Liang Yang, Senior Member, IEEE, and Lajos Hanzo, Fellow, IEEE
}

\begin{abstract}
In this paper, we propose and investigate a relaydiversity transmission scheme for the direct-sequence codedivision multiple-access (DS-CDMA) downlink, where each (destination) mobile terminal (MT) is aided by a cluster of relays to achieve the relay diversity. In the considered system, downlink multiuser interference (MUI) is suppressed with the aid of transmitter preprocessing operated at the base station (BS). Two transmitter preprocessing schemes are considered, which are operated in the principles of transmitter zero forcing (TZF) and transmitter minimum mean square error (TMMSE). At the MTs, signals received from the $B S$ and relays are combined based on the principles of maximal ratio combining (MRC) or maximum signal-to-interference-plus-noise ratio (MSINR). In this paper, the bit-error-rate (BER) performance of the relay-assisted DS-CDMA downlink is investigated when the communication channels are assumed to experience both propagation path loss and generalized Nakagami- $m$ fading. Our study and simulation results show that the transmitter preprocessing can help to achieve the relay diversity by efficiently mitigating the MUI present at the relays and MTs. Furthermore, in our proposed relay-diversity scheme, the relays only require low-complexity signal processing to forward information to their served MTs.
\end{abstract}

Index Terms-Cooperation, direct-sequence code-division multiple access (DS-CDMA), minimum mean square error (MMSE), power allocation, relay diversity, transmitter preprocessing, zero forcing (ZF).

\section{INTRODUCTION}

$\mathbf{I}$ $\mathrm{T}$ is well known that signals transmitted over wireless channels experience fading, the effect of which can usually be mitigated by the employment of various diversity techniques implemented in the time domain, frequency domain, or space domain or in their joints. Recently, spatial diversity achieved by using multiple transmit/receive antennas has drawn wide attention in the research community and industry [1]-[6]. Specifically, where downlink transmission in cellular communications is concerned, transmit diversity may be achieved by deploying multiple transmit antennas at the base station (BS) with the aid

Manuscript received August 20, 2008; revised February 11, 2009 and April 4, 2009. First published April 24, 2009; current version published October 2, 2009. This work was supported by the Engineering and Physical Sciences Research Council of the U.K. The review of this paper was coordinated by Prof. H.-C. Wu.

The authors are with the School of Electronics and Computer Science, University of Southampton, SO17 1BJ Southampton, U.K. (e-mail: wf04r@ecs. soton.ac.uk; 1ly@ecs.soton.ac.uk; lh@ecs.soton.ac.uk).

Digital Object Identifier 10.1109/TVT.2009.2021598 of a certain transmit diversity scheme such as space-time coding [1]-[3] and space-time spreading [7], [8]. Although deploying multiple antennas at the BS is capable of achieving the transmit diversity, this type of transmission schemes does not make use of the nonlinear relationship between the propagation path loss and the propagation distance, as shown in [9]. In practice, when the distance from the BS to an MT is long, intermediate relays may be used to divide the long transmitter-receiver (T-R) path into several relatively short propagation paths to reduce the overall path loss by exploiting the nonlinear relationship between the propagation path loss and the propagation distance. Furthermore, the intermediate relays can be viewed as extra transmit antennas, which can be utilized to attain relay diversity [10]-[14].

In this paper, we propose and investigate a cooperation scheme for direct-sequence code-division multiple-access (DSCDMA) downlink. In our proposed cooperation scheme, a cluster of relays near a destination mobile terminal (MT) is employed to enhance the transmission between the BS and the destination MT. The cooperation scheme is operated based on the time-division mode, where each symbol duration is divided into two time slots. Within the first time slot, the BS broadcasts signals to the destination MTs, as well as to their relays. Within the second time slot, the relays forward the signals received from the BS within the first time slot to the MTs. Since there exists multiuser interference (MUI) within the first time slot and interrelay interference within the second time slot, in the proposed cooperative DS-CDMA downlink, the MUI is suppressed with the aid of transmitter preprocessing [4], [15]-[22] operated at the BS, while the interrelay interference is mitigated using receiver processing carried out at the destination MTs. Therefore, in the proposed cooperative DS-CDMA downlink, the destination MTs and, particularly, the relays may have low complexity due to the employment of transmitter preprocessing executed at the BS. To be more specific, in this paper, the transmitter preprocessing is carried out based on either the zero-forcing (ZF) [4], [15], [17]-[19] or the minimum-mean-square-error (MMSE) [4], [17], [20][22] principles. To make the cooperative DS-CDMA downlink system as simple as possible, we assume that the BS carries out the transmitter preprocessing using only the knowledge about the spreading codes assigned to the destination MTs, as in [16]. We assume that there is no information exchange 


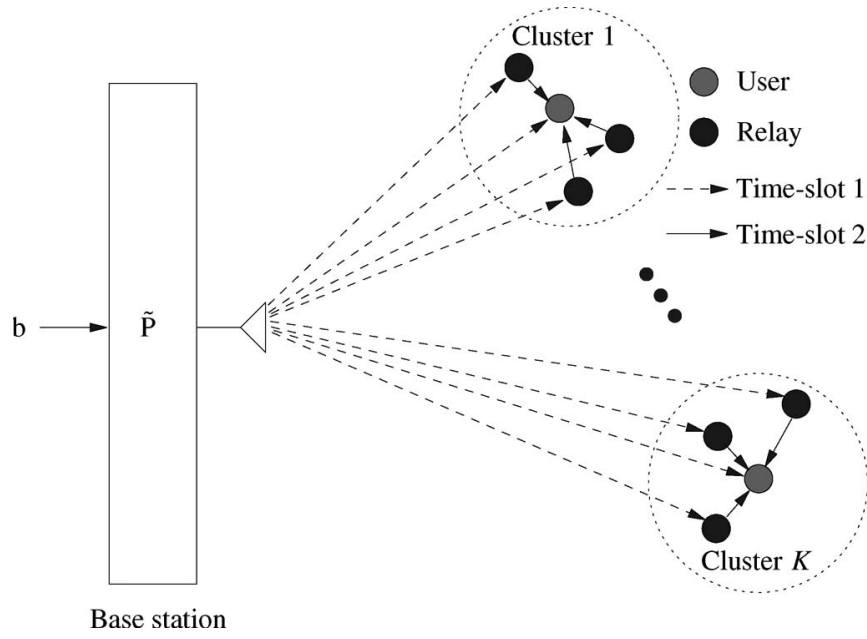

Fig. 1. Schema of the relay-aided DS-CDMA downlink system, which supports $K$ mobile terminals and employs transmitter preprocessing at the BS. Each MT and its $L$ relays form a cluster.

between the BS and any of the relays and that there is also no feedback channel from a destination MT to the BS, implying that the transmitter preprocessing is independent of the channel state information about the channels from the BS to the destination MTs. Finally, at the destination MTs, the transmitted information is detected based on either the maximal-ratiocombining-assisted [23] single-user combining (MRC-SUC) or the maximum signal-to-interference-plus-noise ratio [24], [25] assisted multiuser combining (MSINR-MUC). Furthermore, in this paper, the bit-error-rate (BER) performance of the relayaided DS-CDMA downlink using transmitter preprocessing is investigated, assuming that the communication channels experience both propagation path loss and fast fading [26]. Our study and simulation results show that the proposed cooperative DS-CDMA downlink transmission scheme is capable of efficiently mitigating the MUI and interrelay interference and achieving the relay diversity, as promised.

The remainder of this paper is organized as follows. In Section II, we describe the cooperative DS-CDMA downlink system in terms of the transmitted signal, transmitter preprocessing, and channel model. In Section III, the signal processing at the relays is addressed. Section IV derives the presentation of received signals at the MTs, while Section V considers the detection schemes used by the MTs. Power allocation is considered in Section VI, and simulation results are provided in Section VII. Finally, in Section VIII, the conclusions are summarized.

\section{SYSTEM DESCRIPTION}

We consider a cooperative multiuser DS-CDMA downlink system, as shown in Fig. 1, which supports $K$ MTs (users). Each of the $K$ MTs is aided by $L$ relays. Furthermore, we assume that an MT and its $L$ relays are close to each other and form a cluster. For the sake of clarity of discussion, we refer to the direct channels from the BS to the $K$ MTs as the $\mathrm{D}$-channels. The relay channels from the BS through relays to the $K$ MTs are referred to as the R-channels. Furthermore, the $\mathrm{R}$-channels are divided into the BR-channels and RM-channels.
The BR-channels denote the channels from the BS to the relays, while the RM-channels denote the channels from the relays to the $K$ MTs.

We assume that the cooperation is operated based on the time-division principles. To be more specific, we assume that each symbol duration is divided into two time slots, with their duration equaling $T_{b}$ of the bit duration. Within the first time slot, the BS broadcasts the sum of $K$ user signals to the $K$ destination MTs and also to their $K L$ relays. Within the second time slot, the $K L$ relays forward the signals received from the BS within the first time slot to the $K$ destination MTs. To focus our attention on the relay diversity as well as for the sake of simplicity, in this paper, we assume that any two clusters are sufficiently separated and that, during the second time slot, the interference between any two clusters can be ignored after taking into account the long-distance resulting propagation path loss. Note, however, that our study in this paper may straightforwardly be extended to the communication scenarios, where interference exists among the clusters during the second time slot.

\section{A. Transmission Scheme at the BS}

The signals transmitted by the BS to relays and MTs within the first time slot of a symbol duration are preprocessed signals. Let $s=\left[s_{0}, s_{1}, \ldots, s_{N-1}\right]^{T}$ represent the discrete-time signals transmitted by the BS, where $N$ denotes the number of chips per bit or the spreading factor of the DS-CDMA scheme. When both spreading and transmitter preprocessing are considered, $s$ can be formed as [19], [22], [27]

$$
\boldsymbol{s}=\tilde{\boldsymbol{P}} \boldsymbol{A} \boldsymbol{b}
$$

where $\tilde{\boldsymbol{P}}=\boldsymbol{P} \boldsymbol{C}$, with $\boldsymbol{P}$ being an $(N \times N)$ transmitter preprocessing matrix and $\boldsymbol{C}$ being an $(N \times K)$ spreading matrix structured by the spreading sequences assigned to the $K$ MTs. Explicitly, the transmitter preprocessing and spreading can jointly be implemented by directly determining the matrix $\tilde{\boldsymbol{P}}$, which can be expressed in terms of the $K$ downlink MTs as

$$
\tilde{\boldsymbol{P}}=\left[\tilde{\boldsymbol{p}}_{1}, \tilde{\boldsymbol{p}}_{2}, \ldots, \tilde{\boldsymbol{p}}_{K}\right]
$$

where $\tilde{\boldsymbol{p}}_{k}$ is an $N$-length vector for preprocessing the data to be transmitted to MT $k$. In (1), $\boldsymbol{A}$ is a $(K \times K)$ diagonal matrix expressed as

$$
\boldsymbol{A}=\operatorname{diag}\left\{\sqrt{2 P_{1 t}}, \sqrt{2 P_{2 t}}, \ldots, \sqrt{2 P_{K t}}\right\}
$$

where $P_{k t}$ denotes the transmission power in terms of MT $k$. Finally, in (1), $\boldsymbol{b}$ denotes a $K$-length vector containing the data symbols to be transmitted to the $K$ MTs, which is expressed as

$$
\boldsymbol{b}=\left[b_{1}[n], b_{2}[n], \ldots, b_{K}[n]\right]^{T}, \quad n=0,1, \ldots
$$

where $b_{k}[n]$ is assumed to be binary and takes a value in $\{+1,-1\}$, implying that the binary-phase-shift-keying baseband modulation is assumed. 
Based on (1), the signal broadcast by the BS can be expressed as

$$
s(t)=\sum_{k=1}^{K} \sqrt{2 P_{k t}} b_{k}(t) \tilde{p}_{k}(t) \cos \left(2 \pi f_{c} t\right) .
$$

In (5), $f_{c}$ represents the carrier frequency, $b_{k}(t)=$ $\sum_{n=0}^{\infty} b_{k}[n] P_{T_{b}}\left(t-n T_{b}\right)$ represents the transmitted data waveform, where $P_{T_{b}}(t)$ represents the rectangular waveform defined as $P_{T_{b}}(t)=1$ if $0 \leq t<T_{b}$ and $P_{T_{b}}(t)=0$ otherwise, $\tilde{p}_{k}(t)$ is the waveform formed by $\tilde{\boldsymbol{p}}_{k}$ as shown in (2), and $p_{k}(t)$ can be expressed as $\tilde{p}_{k}(t)=\sum_{n=0}^{\infty} \tilde{p}_{k n} \psi_{T_{c}}\left(t-n T_{c}\right)$, where $\tilde{p}_{k n}$ denotes the $n$th element of $\tilde{\boldsymbol{p}}_{k}, T_{c}$ denotes the chip duration, and $\psi_{T_{c}}(t)$ is the chip waveform defined within $\left[0, T_{c}\right)$ and normalized to satisfy $\int_{0}^{T_{c}} \psi_{T_{c}}^{2}(t) d t=T_{c}$.

Let us assume that the downlink channels experience both propagation path loss and flat fading. Then, it can be shown that the normalized discrete observation vector obtained at the $k$ th MT can be expressed as

$$
\boldsymbol{r}_{k}=\xi_{0}^{(k)} h_{0}^{(k)} \tilde{\boldsymbol{P}} \boldsymbol{A} \boldsymbol{b}+\boldsymbol{n}_{k}, \quad k=1,2, \ldots, K
$$

where $\xi_{0}^{(k)}$ and $h_{0}^{(k)}$ account for the propagation path loss and fast fading of the $k$ th D-channel from the BS to MT $k$, and $\boldsymbol{n}_{k}$ is an $N$-length Gaussian noise vector, which obeys the multivariate Gaussian distribution with zero mean and a covariance matrix of $2 \sigma^{2} \boldsymbol{I}_{N}$, where $\sigma^{2}=N_{0} / 2$, with $N_{0}$ representing the single-sided power spectral density (PSD) of the Gaussian noise process, and $\boldsymbol{I}_{N}$ denotes an $(N \times N)$ identity matrix.

At MT $k, \boldsymbol{r}_{k}$ is despread using the $k$ th MT's spreading sequence $c_{k}$, yielding an observation variable for $b_{k}[n]$, which is expressed as

$$
y_{k}=\boldsymbol{c}_{k}^{T} \boldsymbol{r}_{k}=\xi_{0}^{(k)} h_{0}^{(k)} \boldsymbol{c}_{k}^{T} \tilde{\boldsymbol{P}} \boldsymbol{A} \boldsymbol{b}+n_{k}, \quad k=1,2, \ldots, K
$$

where $n_{k}=\boldsymbol{c}_{k}^{T} \boldsymbol{n}_{k}$, which is still a Gaussian-distributed random variable with zero mean and a variance of $\sigma^{2}$ per dimension.

Let $\boldsymbol{y}=\left[y_{1}, y_{2}, \ldots, y_{K}\right]^{T}$. Then, it can be shown that $\boldsymbol{y}$ can be expressed as

$$
\boldsymbol{y}=\boldsymbol{\xi} \boldsymbol{H} \boldsymbol{C}^{T} \tilde{\boldsymbol{P}} \boldsymbol{A} \boldsymbol{b}+\boldsymbol{n}
$$

where by definition, we have

$$
\begin{aligned}
\boldsymbol{\xi} & =\operatorname{diag}\left\{\xi_{0}^{(1)}, \xi_{0}^{(2)}, \ldots, \xi_{0}^{(K)}\right\} \\
\boldsymbol{H} & =\operatorname{diag}\left\{h_{0}^{(1)}, h_{0}^{(2)}, \ldots, h_{0}^{(K)}\right\} \\
\boldsymbol{n} & =\left[n_{1}, n_{2}, \ldots, n_{K}\right]^{T} .
\end{aligned}
$$

As shown in (8), there exists interference among the $K$ downlink MTs, when $\boldsymbol{C}^{T} \tilde{\boldsymbol{P}}$ is not a diagonal matrix. In this case, transmitter preprocessing [4], [15], [17]-[22] may be employed to suppress the downlink MUI. Let us derive below the preprocessing matrix $\tilde{\boldsymbol{P}}$, when the transmitter preprocessing is based on the principles of either ZF or MMSE. Note that, in this paper, the preprocessing is carried out under the following assumptions.

1) The BS employs the knowledge about the spreading sequences assigned to the $K$ MTs but does not have the knowledge about the downlink channels associated with the $K$ MTs.

2) There is no information exchange between the BS and any of the $K L$ relays.

1) $T Z F$ : The transmitter preprocessing based on the $\mathrm{ZF}$ principles, which we refer to as the transmitter ZF (TZF), is capable of fully removing the downlink MUI. Based on (8) and the above-stated assumptions for preprocessing, the preprocessing matrix for the TZF can be expressed as [15], [17]-[19]

$$
\tilde{\boldsymbol{P}}=\beta \boldsymbol{C}\left(\boldsymbol{C}^{T} \boldsymbol{C}\right)^{-1} .
$$

When applying (10) into (8), we can obtain $\boldsymbol{y}=\beta \boldsymbol{\xi} \boldsymbol{H} \boldsymbol{A} \boldsymbol{b}+\boldsymbol{n}$. Explicitly, the MUI existing among the downlink MTs is fully removed, since $\boldsymbol{\xi}, \boldsymbol{H}$, and $\boldsymbol{A}$ are all diagonal matrices. However, as the ZF multiuser detection [24], the TZF-assisted transmitter preprocessing eliminates MUI at the cost of background noise amplification [20].

In (10), the parameter $\beta$ is applied to achieve the constraint on the transmission power. The value of $\beta$ can be determined according to $E\left[\|\tilde{\boldsymbol{P}} \boldsymbol{b}\|^{2}\right]=E\left[\|\boldsymbol{b}\|^{2}\right]$, which yields [4], [17], [19]

$$
\beta=\sqrt{\frac{K}{\operatorname{trace}\left(\left(\boldsymbol{C}^{T} \boldsymbol{C}\right)^{-1}\right)}}
$$

where trace $(\boldsymbol{A})$ denotes the trace of the square matrix $\boldsymbol{A}$.

2) TMMSE: The transmitter preprocessing based on the MMSE principles, which is referred to as the transmitter MMSE (TMMSE), is capable of mitigating the downlink MUI while simultaneously suppressing the background noise [17], [20]-[22]. In the context of the TMMSE, based on (8), and the available knowledge for preprocessing, the transmitter preprocessing matrix can be expressed as [4], [17], [22]

$$
\tilde{\boldsymbol{P}}=\beta \boldsymbol{C}\left(\boldsymbol{C}^{T} \boldsymbol{C}+2 \sigma^{2} \boldsymbol{\rho}\right)^{-1}
$$

where $\boldsymbol{\rho}=\operatorname{diag}\left\{\rho_{1}, \rho_{2}, \ldots, \rho_{K}\right\} \quad$ contains the noisesuppression factors [22] with respect to the $K$ MTs. Note that the noise-suppression factors may be optimized to achieve the best possible performance when ideal knowledge about the noise power associated with the $K$ MTs is not reliable. As our simulation results in Section VII shows, when the BS has no knowledge about the noise power of the $K$ MTs, the matrix $\rho$ in (12) may be set to an appropriate nonzero diagonal matrix. As a result, the performance achieved in this case may still outperform that achieved by the DS-CDMA downlink employing the TZF-assisted transmitter preprocessing.

In (12), the parameter $\beta$ for achieving the power constraint can be evaluated by

$$
\beta=\sqrt{\frac{K}{\operatorname{trace}\left(\hat{\boldsymbol{P}} \hat{\boldsymbol{P}}^{H}\right)}}
$$


where by definition, we have $\hat{\boldsymbol{P}}=\boldsymbol{C}\left(\boldsymbol{C}^{T} \boldsymbol{C}+2 \sigma^{2} \boldsymbol{\rho}\right)^{-1}$. Let us now consider the channel model.

\section{B. Channel Model}

There are three types of channels, namely, the D-channels, BR-channels, and RM-channels, associated with the considered relay-assisted DS-CDMA downlink systems. We assume that the downlink channels experience both propagation path loss and fast fading. We assume that the propagation path loss $L_{p}(d)$ can be expressed with respect to the T-R distance $d$ as [26]

$$
L_{p}(d)(\mathrm{dB})=L_{s}\left(d_{0}\right)(\mathrm{dB})+10 \eta \log \left(\frac{d}{d_{0}}\right)
$$

where $L_{s}\left(d_{0}\right)$ denotes the path loss measured at the reference distance $d_{0}$, and $\eta$ is the path-loss exponent, which takes a typical value of $\eta=2$ in free space and $\eta=4$ in cellular mobile systems [26].

The fast fading experienced by the downlink signals transmitted over the D-channels, BR-channels, and the RM-channels is modeled by the generalized Nakagami- $m$ fading. In detail, let the (fast-)fading gains of the D-channels be expressed as $\left\{h_{0}^{(k)}=\alpha_{00}^{(k)} e^{j \theta_{00}^{(k)}}, k=1, \ldots, K\right\}$, those of the BR-channels be expressed as $\left\{h_{l}^{(k)}=\alpha_{l 1}^{(k)} e^{j \theta_{l 1}^{(k)}}, \quad l=1, \ldots, L ; \quad k=\right.$ $1, \ldots, K\}$, and those of the RM-channels be expressed as $\left\{h_{r l}^{(k)}=\alpha_{l 2}^{(k)} e^{j \theta_{l 2}^{(k)}}, l=1, \ldots, L ; k=1, \ldots, K\right\}$. Then, the phases $\left\{\theta_{l i}^{(k)}\right\}$ are assumed to obey the independent uniform distribution in $[0,2 \pi)$, while $\left\{\alpha_{l i}^{(k)}\right\}$ obey the Nakagami- $m$ distribution with the probability density function given by [28]

$$
\begin{aligned}
f_{\alpha_{l i}^{(k)}}(y) & =\frac{2 m_{l i}^{m_{l i}} y^{2 m_{l i}-1}}{\Gamma\left(m_{l i}\right) \Omega_{l i}} \exp \left(-\frac{m_{l i}}{\Omega_{l i}} y^{2}\right), \quad i=0,1,2 \\
l & =0,1, \ldots, L ; \quad k=1,2, \ldots, K
\end{aligned}
$$

where $m_{l i}$ represents the fading parameter of the D-channels, BR-channels, or RM-channels, depending on the values of $l$ and $i$, and $\Omega_{l i}=E\left[\left(\alpha_{l i}^{(k)}\right)^{2}\right]$. As shown in (15), the parameters $m_{l i}$ and $\Omega_{l i}$ are independent of the index $k$, implying that all the downlink signals with respect to the $K$ MTs are assumed to experience identical fading.

Note that we use the generalized Nakagami- $m$ distribution as shown in (15) since in the considered relay-assisted DSCDMA downlink systems, the D-channels, the BR-channels, and the RM-channels may experience different fast fading. Specifically, the distance between a relay and its assisted MT may significantly be shorter than the distance between the BS and the relay or shorter than that between the BS and the MT. In this case, the corresponding RM-channel may experience less severe fading than the D-channels or the BR-channels. Correspondingly, we may model the RM-channels by the Nakagami$m$ fading associated with a relatively high $m$ value and model the D-channels or BR-channels by the Nakagami- $m$ fading associated with a relatively low $m$ value. For example, we can assume that the RM-channels experience the Nakagami- $m$ fading associated with a value of $m>1$, and the D-channels and the BR-channels experience the Rayleigh fading, which corresponds to the Nakagami- $m$ fading with $m=1$.

\section{Signal PRocessing AND ForWARding AT RELAYS}

The relays receive and process signals transmitted by the BS within the first time slot of a symbol duration. Within the second time slot of a symbol duration, the processed signals are forwarded by the relays to their served MTs. In this section, we consider the operations carried out at the relays.

When the downlink DS-CDMA signals in the form of (5) are transmitted over flat-fading channels, the complex baseband equivalent signal received by the $l$ th relay of the $k$ th MT within the first time slot of the $n$th symbol duration can be written as

$$
\begin{aligned}
r_{l}^{(k)}(t) & =h_{l}^{(k)} \sum_{k^{\prime}=1}^{K} \sqrt{2 P_{k^{\prime} l}^{(k)}} b_{k^{\prime}}[n] \tilde{p}_{k^{\prime}}(t)+n_{l}^{(k)}(t) \\
l & =1,2, \ldots, L ; \quad k=1,2, \ldots, K
\end{aligned}
$$

where $P_{k^{\prime} l}^{(k)}$ represents the power received by the $l$ th relay of MT $k$ from the $k^{\prime}$ th user signal transmitted by the BS after taking into account the path loss of the BR-channel, and $h_{l}^{(k)}$ represents the fading gain of the BR-channel from the BS to the $l$ th relay of MT $k$, while $n_{l}^{(k)}(t)$ denotes the Gaussian noise observed at the $l$ th relay of MT $k$, which has mean zero and a single-sided PSD of $N_{0}$ per dimension.

Let us express the observation and noise samples obtained at the $l$ th relay of MT $k$ as

$$
\begin{aligned}
\boldsymbol{y}_{l}^{(k)} & =\left[y_{l 0}^{(k)}, y_{l 1}^{(k)}, \ldots, y_{l(N-1)}^{(k)}\right]^{T} \\
\tilde{\boldsymbol{n}}_{l}^{(k)} & =\left[\tilde{n}_{l 0}^{(k)}, \tilde{n}_{l 1}^{(k)}, \ldots, \tilde{n}_{l(N-1)}^{(k)}\right]^{T}
\end{aligned}
$$

where $\tilde{\boldsymbol{n}}_{l}^{(k)}$ is a Gaussian noise vector distributed with mean zero and a covariance matrix $N_{0} / E_{l}^{(k)} \boldsymbol{I}_{N}$, where $E_{l}^{(k)}=$ $P_{k l}^{(k)} T_{b}$. Then, it can be shown that $\boldsymbol{y}_{l}^{(k)}$ can be expressed as

$$
\begin{aligned}
\boldsymbol{y}_{l}^{(k)} & =h_{l}^{(k)} \tilde{\boldsymbol{p}}_{k} b_{k}[n]+h_{l}^{(k)} \sum_{k^{\prime} \neq k}^{K} \sqrt{\frac{P_{k^{\prime} l}^{(k)}}{P_{k l}^{(k)}}} \tilde{\boldsymbol{p}}_{k^{\prime}} b_{k^{\prime}}[n]+\tilde{\boldsymbol{n}}_{l}^{(k)} \\
l & =1,2, \ldots, L .
\end{aligned}
$$

Let us assume that the $l$ th relay of MT $k$ employs the knowledge of $\boldsymbol{c}_{k}$ of the spreading sequence assigned to the $k$ th MT. We also assume that the $l$ th relay of MT $k$ employs the knowledge of $h_{l}^{(k)}$ of the channel gain from the BS to this relay. Note that the channel gain $h_{l}^{(k)}$ may be estimated in the same way as estimating the channel from the BS to MT $k$, for example, with the aid of the pilot information sent by the BS to the $k$ th MT. Then, the $l$ th relay of MT $k$ can estimate $b_{k}[n]$ by forming the soft-decision variable of

$$
\begin{aligned}
\hat{b}_{l}^{(k)}[n] & =\frac{1}{\left|h_{l}^{(k)}\right|^{2}}\left(h_{l}^{(k)}\right)^{*} \boldsymbol{c}_{k}^{T} \boldsymbol{y}_{l}^{(k)} \\
& =\boldsymbol{c}_{k}^{T} \tilde{\boldsymbol{p}}_{k} b_{k}[n]+\sum_{k^{\prime} \neq k}^{K} \sqrt{\frac{P_{k^{\prime} l}^{(k)}}{P_{k l}^{(k)}}} \boldsymbol{c}_{k}^{T} \tilde{\boldsymbol{p}}_{k^{\prime}} b_{k^{\prime}}[n]+\frac{1}{h_{l}^{(k)}} \boldsymbol{c}_{k}^{T} \tilde{\boldsymbol{n}}_{l}^{(k)}
\end{aligned}
$$


After the estimation, $\hat{b}_{l}^{(k)}[n]$ of (19) is then respread and forwarded by the $l$ th relay to MT $k$ using the second time slot of the $n$th symbol duration. The transmitted signal of the $l$ th relay of MT $k$ can be expressed as

$$
s_{l}^{(k)}(t)=\sqrt{\frac{2 P_{l t}^{(k)}}{\varsigma_{k l}}} \hat{b}_{l}^{(k)}[n] c_{l}^{(k)}(t) \cos \left(2 \pi f_{c} t+\phi_{l}^{(k)}\right)
$$

where $l=1,2, \ldots, L$, and $P_{l t}^{(k)}, c_{l}^{(k)}(t), f_{c}$, and $\phi_{l}^{(k)}$ represent, respectively, the transmission power, signature waveform, carrier frequency, and initial phase associated with the $l$ th relay of MT $k$. In (20), $\varsigma_{k l}$ is a normalization coefficient applied so that the transmission power of $s_{l}^{(k)}(t)$ is $P_{l t}^{(k)} ; \varsigma_{k l}$ can be evaluated by

$$
\begin{aligned}
\varsigma_{k l} & =\mathrm{E}\left[\left|\hat{b}_{l}^{(k)}[n]\right|^{2}\right] \\
& =\sum_{k^{\prime}=1}^{K} \frac{P_{k^{\prime}}^{(k)}}{P_{k l}^{(k)}} \boldsymbol{c}_{k}^{T} \tilde{\boldsymbol{p}}_{k^{\prime}} \tilde{\boldsymbol{p}}_{k^{\prime}}^{T} \boldsymbol{c}_{k}+\frac{1}{\left|h_{l}^{(k)}\right|^{2}} \frac{N_{0}}{E_{l}^{(k)}} .
\end{aligned}
$$

Note that it can be shown that, when the TZF is applied, we have, respectively

$$
\begin{aligned}
\hat{b}_{l}^{(k)}[n] & =\beta b_{k}[n]+\frac{1}{h_{l}^{(k)}} \boldsymbol{c}_{k}^{T} \tilde{\boldsymbol{n}}_{l}^{(k)} \\
\varsigma_{k l} & =\beta^{2}+\frac{1}{\left|h_{l}^{(k)}\right|^{2}} \frac{N_{0}}{E_{l}^{(k)}} .
\end{aligned}
$$

Let us now consider the signals received at MT $k$.

\section{REPRESENTATION OF RECEIVED SignAls AT Mobile Terminals}

The MTs receive signals from both the first and second time slots of a symbol duration. Within the first time slot of a symbol duration, the MTs receive signals from the BS. Specifically, the received complex baseband equivalent signal by MT $k$ within the first time slot of the $n$th symbol duration can be expressed as

$$
\begin{aligned}
r_{0}^{(k)}(t) & =h_{0}^{(k)} \sum_{k^{\prime}=1}^{K} \sqrt{2 P_{k^{\prime} r}^{(k)}} b_{k^{\prime}}[n] \tilde{p}_{k^{\prime}}(t)+n(t) \\
k & =1,2, \ldots, K
\end{aligned}
$$

where $P_{k^{\prime} r}^{(k)}, k^{\prime}=1, \ldots, K$ represents the power received by MT $k$ from the $k^{\prime}$ th signal transmitted by the BS after taking into account the path loss of the D-channel from the BS to MT $k$. In (23), $h_{0}^{(k)}$ represents the fading gain accounting for the fast fading of the $k$ th $\mathrm{D}$-channel, while $n(t)$ denotes the complex baseband equivalent Gaussian noise at MT $k$, which has mean zero and a single-sided PSD of $N_{0}$ per dimension.

Within the second time slot of a symbol duration, the MTs receive signals from their relays. We assume that the relays serving a given MT have similar distances from the MT and experience the same large-scale fading. However, we assume that the relays of a given MT are also sufficiently separated, resulting in that the signals received by the MT from its relays experience independent small-scale fading. Under these assumptions, the received complex baseband equivalent signal by MT $k$ during the second time slot of the $n$th symbol duration can, hence, be expressed as

$$
\begin{aligned}
r_{1}^{(k)}(t) & =\sum_{l=1}^{L} \sqrt{\frac{2 P_{r}^{(k)}}{\varsigma_{k l}}} h_{r l}^{(k)} \hat{b}_{l}^{(k)}[n] c_{l}^{(k)}(t)+n(t) \\
k & =1,2, \ldots, K
\end{aligned}
$$

where $P_{r}^{(k)}$ represents the received power of MT $k$ from any one of its $L$ relays, while $h_{r l}^{(k)}$ denotes the (fast-)fading gain of the $l$ th RM-channel of MT $k$.

Let, after the chip-waveform matched filtering, the observations obtained from the first and second time slots of the $n$th symbol duration be collected into $\boldsymbol{y}_{0}^{(k)}$ and $\boldsymbol{y}_{1}^{(k)}$, where $\boldsymbol{y}_{i}^{(k)}=\left[y_{i 0}^{(k)}, y_{i 1}^{(k)}, \ldots, y_{i(N-1)}^{(k)}\right]^{T}, i=0,1$. Then, after the normalization by $\sqrt{2 P_{k r}^{(k)} N} T_{c}$, it can be shown that $\boldsymbol{y}_{0}^{(k)}$ can be expressed as

$$
\begin{aligned}
\boldsymbol{y}_{0}^{(k)} & =h_{0}^{(k)} \tilde{\boldsymbol{p}}_{k} b_{k}[n]+h_{0}^{(k)} \sum_{k^{\prime} \neq k}^{K} \sqrt{\frac{P_{k^{\prime} r}^{(k)}}{P_{k r}^{(k)}}} \tilde{\boldsymbol{p}}_{k^{\prime}} b_{k^{\prime}}[n]+\tilde{\boldsymbol{n}}_{0} \\
k & =1,2, \ldots, K
\end{aligned}
$$

where $\tilde{\boldsymbol{n}}_{0}=\left[\tilde{n}_{00}, \tilde{n}_{01}, \ldots, \tilde{n}_{0(N-1)}\right]^{T}$ is an $N$-length Gaussian noise vector distributed with mean zero and a covariance matrix of $N_{0} / E_{0}^{(k)} \boldsymbol{I}_{N}$, where $E_{0}^{(k)}=P_{k r}^{(k)} T_{b}$ represents the energy per bit received by the $k$ th MT from the BS.

Similarly, after normalization using $\sqrt{2 P_{r}^{(k)} N} T_{c}$, we can express the $\boldsymbol{y}_{1}^{(k)}$ of the observations obtained from the second time slot as

$$
\begin{aligned}
\boldsymbol{y}_{1}^{(k)}=\sum_{l=1}^{L} & \boldsymbol{c}_{l}^{(k)} \sqrt{\frac{1}{\varsigma_{k l}}} h_{r l}^{(k)} \boldsymbol{c}_{k}^{T} \tilde{\boldsymbol{p}}_{k} b_{k}[n] \\
& +\sum_{l=1}^{L} \boldsymbol{c}_{l}^{(k)} \sqrt{\frac{1}{\varsigma_{k l}}} h_{r l}^{(k)} \sum_{k^{\prime} \neq k}^{K} \sqrt{\frac{P_{k^{\prime}, l}^{(k)}}{P_{k, l}^{(k)}}} \boldsymbol{c}_{k}^{T} \tilde{\boldsymbol{p}}_{k^{\prime}} b_{k^{\prime}}[n] \\
& +\sum_{l=1}^{L} \boldsymbol{c}_{l}^{(k)} \sqrt{\frac{1}{\varsigma_{k l}}} h_{r l}^{(k)}\left[\frac{\boldsymbol{c}_{k}^{T} \tilde{\boldsymbol{n}}_{l}^{(k)}}{h_{l}^{(k)}}\right]+\tilde{\boldsymbol{n}}_{1}
\end{aligned}
$$

where $\tilde{\boldsymbol{n}}_{1}$ represents the $N$-length Gaussian noise vector, which has mean zero and a covariance matrix $N_{0} / E_{r}^{(k)} \boldsymbol{I}_{N}$, where $E_{r}^{(k)}=P_{r}^{(k)} T_{b}$ denotes the energy per bit received by the $k$ th MT from its $l$ th relay.

Alternatively, (26) can be expressed in a compact form as

$$
\boldsymbol{y}_{1}^{(k)}=\boldsymbol{C}_{k} \boldsymbol{A}_{k} \boldsymbol{h}_{k} \boldsymbol{c}_{k}^{T} \tilde{\boldsymbol{p}}_{k} b_{k}[n]+\boldsymbol{n}_{I}
$$

associated with defining

$$
\begin{aligned}
\boldsymbol{n}_{I}=\boldsymbol{C}_{k} \boldsymbol{A}_{k} \boldsymbol{h}_{k} \sum_{k^{\prime} \neq k}^{K} & \sqrt{\frac{P_{k^{\prime}, l}^{(k)}}{P_{k, l}^{(k)}} \boldsymbol{c}_{k}^{T} \tilde{\boldsymbol{p}}_{k^{\prime}} b_{k^{\prime}}[n]} \\
& +\boldsymbol{C}_{k} \boldsymbol{A}_{k} \boldsymbol{H}_{k}\left(\boldsymbol{I}_{L} \otimes \boldsymbol{c}_{k}^{T}\right) \tilde{\boldsymbol{n}}^{(k)}+\tilde{\boldsymbol{n}}_{1}
\end{aligned}
$$


where $\otimes$ denotes the Kronecker product [25], and the related matrices and vectors are defined as follows.

1) $C_{k}$ is an $(N \times L)$ matrix, which can be expressed as

$$
\boldsymbol{C}_{k}=\left[\boldsymbol{c}_{1}^{(k)}, \boldsymbol{c}_{2}^{(k)}, \ldots, \boldsymbol{c}_{L}^{(k)}\right]
$$

where $\boldsymbol{c}_{l}^{(k)}, l=1, \ldots, L$ is the spreading sequence used by the $l$ th relay of MT $k$.

2) $\boldsymbol{A}_{k}$ is an $(L \times L)$ matrix given by

$$
\boldsymbol{A}_{k}=\operatorname{diag}\left\{\sqrt{\frac{1}{\varsigma_{k 1}}}, \sqrt{\frac{1}{\varsigma_{k 2}}}, \ldots, \sqrt{\frac{1}{\varsigma_{k L}}}\right\}
$$

where the normalization coefficients $\varsigma_{k l}$, for $l=1, \ldots, L$, are given in (21).

3) $\boldsymbol{h}_{k}$ is an $L$-length vector related to the RM-channels of MT $k$, which can be expressed as

$$
\boldsymbol{h}_{k}=\left[h_{r 1}^{(k)}, h_{r 2}^{(k)}, \ldots, h_{r L}^{(k)}\right]^{T} .
$$

4) $\boldsymbol{H}_{k}$ is an $(L \times L)$ matrix related to both the BR-channels and the RM-channels, which can be expressed as

$$
\boldsymbol{H}_{k}=\operatorname{diag}\left\{\frac{h_{r 1}^{(k)}}{h_{1}^{(k)}}, \frac{h_{r 2}^{(k)}}{h_{2}^{(k)}}, \ldots, \frac{h_{r L}^{(k)}}{h_{L}^{(k)}}\right\} .
$$

5) Finally, $\tilde{\boldsymbol{n}}^{(k)}$ is a Gaussian noise vector of length $L N$, which can be expressed as

$$
\tilde{\boldsymbol{n}}^{(k)}=\left[\left(\tilde{\boldsymbol{n}}_{1}^{(k)}\right)^{T},\left(\tilde{\boldsymbol{n}}_{2}^{(k)}\right)^{T}, \ldots,\left(\tilde{\boldsymbol{n}}_{L}^{(k)}\right)^{T}\right]^{T}
$$

where $\tilde{\boldsymbol{n}}_{l}^{(k)}, l=1, \ldots, L$ is given by (17).

\section{Signal Detection at Mobile Terminals}

Since there is no interference between the first and second time slots, the signals received within the first and second time slots can separately be treated before the final-stage combining for making a decision. Furthermore, since transmitter preprocessing is employed at the BS to suppress the downlink MUI, signals transmitted over the D-channels within the first time slot conflict with no MUI when the TZF is employed or conflict with very low MUI when the TMMSE is employed. By contrast, within the second time slot, the $L$ number of relays simultaneously communicate with the $k$ th MT, and they may interfere with each other. Therefore, the signals received by MT $k$ through its RM-channels during the second time slot need to be combined with considering MUI suppression. In this paper, as two examples, two combining schemes are considered, which are the MRC-SUC and the MSINR-MUC.

Note that the single-user-combining and multiusercombining schemes derived below are suitable for the systems using either the TZF or the TMMSE. If only the TZF is considered, the relevant equations may further be simplified with the aid of (22). Additionally, it is worth noting that, although the TMMSE is capable of simultaneously mitigating both the MUI and the background noise, it, however, cannot fully eliminate the MUI. As our simulation results in Section VII shows, the leaked MUI from the TMMSE may cause performance degradation in the high-SNR region, particularly when random spreading sequences are employed.

The received signal by MT $k$ over the D-channel during the first time slot of the $n$th symbol duration is first despread using $\boldsymbol{c}_{k}^{T}$, yielding

$$
\begin{aligned}
\bar{y}_{0}^{(k)} & =\boldsymbol{c}_{k}^{T} \boldsymbol{y}_{0}^{(k)} \\
& =h_{0}^{(k)} \boldsymbol{c}_{k}^{T} \tilde{\boldsymbol{p}}_{k} b_{k}[n]+\underbrace{h_{0}^{(k)} \sum_{k^{\prime} \neq k}^{K} \sqrt{\frac{P_{k^{\prime} r}^{(k)}}{P_{k r}^{(k)}}} \boldsymbol{c}_{k}^{T} \tilde{\boldsymbol{p}}_{k^{\prime}} b_{k^{\prime}}[n]}_{I_{D}}+\boldsymbol{c}_{k}^{T} \tilde{\boldsymbol{n}}_{0}
\end{aligned}
$$

where $I_{D}$ denotes the downlink MUI within the first time slot, which is zero when the TZF is employed and is usually very small when the TMMSE is employed. Based on (34), it can be shown that, after approximating $I_{D}$ as a Gaussian-distributed random variable, the weight for finally combining $\bar{y}_{0}^{(k)}$ can be expressed as

$$
w_{0}^{(k)}=\left(\frac{N_{0}}{E_{0}^{(k)}}+\sigma_{D}^{2}\right)^{-1} \boldsymbol{c}_{k}^{T} \tilde{\boldsymbol{p}}_{k}\left(h_{0}^{(k)}\right)^{*}
$$

where $\sigma_{D}^{2}$ represents the second-order moment of $I_{D}$, which is given by

$$
\sigma_{D}^{2}=\left|h_{0}^{(k)}\right|^{2} \sum_{k^{\prime} \neq k}^{K} \frac{P_{k^{\prime} r}^{(k)}}{P_{k r}^{(k)}} \boldsymbol{c}_{k}^{T} \tilde{\boldsymbol{p}}_{k^{\prime}} \tilde{\boldsymbol{p}}_{k^{\prime}}^{T} \boldsymbol{c}_{k}
$$

Let us now consider the combining of the signals received by MT $k$ during the second time slot of the $n$th symbol duration.

\section{A. Maximal-Ratio-Combining- \\ Assisted-Single-User Combining}

The signals received within the second time slot of the $n$th symbol duration is $\boldsymbol{y}_{1}^{(k)}$, as given by (27). When the MRCSUC is considered, $\boldsymbol{y}_{1}^{(k)}$ is first despread using the $C_{k}^{T}$ of (29), yielding

$$
\overline{\boldsymbol{y}}=\left[\bar{y}_{1}^{(k)}, \bar{y}_{2}^{(k)}, \ldots, \bar{y}_{L}^{(k)}\right]=\boldsymbol{C}_{k}^{T} \boldsymbol{y}_{1}^{(k)} .
$$

Upon substituting (27) and (29) into (37), the $l$ th entry of $\bar{y}$ can be expressed as

$$
\begin{aligned}
\bar{y}_{l}^{(k)}= & \sqrt{\frac{1}{\varsigma_{k l}}} h_{r l}^{(k)} \boldsymbol{c}_{k}^{T} \tilde{\boldsymbol{p}}_{k} b_{k}[n] \\
& +\sum_{l^{\prime}=1}^{L} \sqrt{\frac{1}{\varsigma_{k l l}}} \frac{h_{r l^{\prime}}^{(k)}}{h_{l^{\prime}}^{(k)}}\left(\boldsymbol{c}_{l}^{(k)}\right)^{T} \boldsymbol{c}_{l^{\prime}}^{(k)} \boldsymbol{c}_{k}^{T} \boldsymbol{n}_{l^{\prime}}^{(k)} \\
& +\left(\boldsymbol{c}_{l}^{(k)}\right)^{T} \boldsymbol{n}_{1}+I_{R}, \quad l=1,2, \ldots, L
\end{aligned}
$$


where $I_{R}$ is given by

$$
\begin{aligned}
I_{R}= & \sum_{l^{\prime} \neq l}^{L} \sqrt{\frac{1}{\varsigma_{k l l}}} h_{r l^{\prime}}^{(k)}\left(\boldsymbol{c}_{l}^{(k)}\right)^{T} \boldsymbol{c}_{l^{\prime}}^{(k)} \boldsymbol{c}_{k}^{T} \tilde{\boldsymbol{p}}_{k} b_{k}[n] \\
& +\sum_{l^{\prime}=1}^{L} \sqrt{\frac{1}{\varsigma_{k l^{\prime}}}} h_{r l^{\prime}}^{(k)}\left(\boldsymbol{c}_{l}^{(k)}\right)^{T} \boldsymbol{c}_{l^{\prime}}^{(k)} \sum_{k^{\prime} \neq k}^{K} \sqrt{\frac{P_{k^{\prime} l}^{(k)}}{P_{k l}^{(k)}}} \boldsymbol{c}_{k}^{T} \tilde{\boldsymbol{p}}_{k^{\prime}} b_{k^{\prime}}[n] .
\end{aligned}
$$

Note that, at the right-hand side of (38), the first term is the desired output, the second term is the noise forwarded by the $L$ relays, the third term is the noise received at MT $k$, and finally, $I_{R}$ is the MUI forwarded by the $L$ relays to MT $k$.

For the MRC-SUC, the $\bar{y}_{l}^{(k)}$ of (38) is approximated as a Gaussian-distributed signal with a mean given by the first term at the right-hand side of (38) and a variance given by

$$
\begin{aligned}
& \sigma_{R}^{2}=\sum_{l^{\prime} \neq l}^{L} \frac{1}{\varsigma_{k l^{\prime}}}\left|h_{r l^{\prime}}^{(k)}\left(\boldsymbol{c}_{l}^{(k)}\right)^{T} \boldsymbol{c}_{l^{\prime}}^{(k)} \boldsymbol{c}_{k}^{T} \tilde{\boldsymbol{p}}_{k}\right|^{2} \\
& +\sum_{l^{\prime}=1}^{L} \frac{1}{\varsigma_{k l^{\prime}}}\left|h_{r l^{\prime}}^{(k)}\left(\boldsymbol{c}_{l}^{(k)}\right)^{T} \boldsymbol{c}_{l^{\prime}}^{(k)}\right|^{2} \sum_{k^{\prime} \neq k}^{K} \frac{P_{k^{\prime} l}^{(k)}}{P_{k l}^{(k)}} \boldsymbol{c}_{k}^{T} \tilde{\boldsymbol{p}}_{k^{\prime}} \tilde{\boldsymbol{p}}_{k^{\prime}}^{T} \boldsymbol{c}_{k} .
\end{aligned}
$$

Consequently, the weights achieving the MRC-SUC can be expressed as [9]

$$
\begin{array}{r}
w_{l}^{(k)}=\left(\sum_{l^{\prime}=1}^{L} \frac{\left|h_{r l^{\prime}}^{(k)}\right|^{2} l^{\prime}\left|h_{l^{\prime}}^{(k)}\right|^{2}}{2}\left|\left(\boldsymbol{c}_{l}^{(k)}\right)^{T} \boldsymbol{c}_{l^{\prime}}^{(k)}\right|^{2} N_{0}+N_{0}+\sigma_{R}^{2}\right)^{-1} \\
\times \sqrt{\frac{1}{\varsigma_{k l}}} \beta\left(h_{r l}^{(k)}\right)^{*}
\end{array}
$$

Finally, when both the first and second time slots of the $n$th symbol duration are considered, the decision variable $z_{k}[n]$ for $b_{k}[n]$ can be formed as

$$
z_{k}[n]=\sum_{l=0}^{L} w_{l}^{(k)} \bar{y}_{l}^{(k)}, \quad k=1,2, \ldots, K .
$$

Let us now consider the MSINR-MUC.

\section{B. MSINR-MUC}

For convenience, we express the observations of (27) as

$$
\boldsymbol{y}_{1}^{(k)}=\overline{\boldsymbol{h}}_{k} b_{k}[n]+\boldsymbol{n}_{I}
$$

where by definition

$$
\begin{aligned}
& \overline{\boldsymbol{h}}_{k}= \boldsymbol{C}_{k} \boldsymbol{A}_{k} \boldsymbol{h}_{k} \boldsymbol{c}_{k}^{T} \tilde{\boldsymbol{p}}_{k} \\
& \boldsymbol{n}_{I}=\boldsymbol{C}_{k} \boldsymbol{A}_{k} \boldsymbol{h}_{k} \sum_{k^{\prime} \neq k}^{K} \sqrt{\frac{P_{k^{\prime} l}^{(k)}}{P_{k l}^{(k)}} \boldsymbol{c}_{k}^{T} \tilde{\boldsymbol{p}}_{k^{\prime}} b_{k^{\prime}}[n]} \\
& \quad+\boldsymbol{C}_{k} \boldsymbol{A}_{k} \boldsymbol{H}_{k}\left(\boldsymbol{I}_{L} \otimes \boldsymbol{c}_{k}^{T}\right) \tilde{\boldsymbol{n}}_{k}+\tilde{\boldsymbol{n}}_{1}
\end{aligned}
$$

where $\overline{\boldsymbol{h}}_{k}$ may be viewed as the equivalent channel impulse response associated with MT $k$, and $\boldsymbol{n}_{I}$ contains the MUI, interrelay interference, and background noise.

Let $\boldsymbol{w}$ be an $N$-length vector for combining $\boldsymbol{y}_{1}^{(k)}$ in the maximum signal-to-interference-plus-noise ratio (MSINR) sense. According to (43), it can be shown that $\boldsymbol{w}$ can be expressed as [9], [25], [29]

$$
\boldsymbol{w}=\mu \boldsymbol{R}_{I}^{-1} \overline{\boldsymbol{h}}_{k}
$$

where $\mu$ denotes a constant, and $\boldsymbol{R}_{I}$ represents the covariance matrix of $\boldsymbol{n}_{I}$, which, after some simplification, can be expressed as

$$
\begin{aligned}
\boldsymbol{R}_{I}= & E\left[\boldsymbol{n}_{I} \boldsymbol{n}_{I}^{H}\right] \\
= & \sum_{l=1}^{L} \frac{1}{\varsigma_{k l}}\left|h_{r l}^{(k)}\right|^{2} \boldsymbol{c}_{l}^{(k)}\left(\boldsymbol{c}_{l}^{(k)}\right)^{T} \sum_{k^{\prime} \neq k}^{K} \frac{P_{k^{\prime} l}^{(k)}}{P_{k l}^{(k)}} \boldsymbol{c}_{k}^{T} \tilde{\boldsymbol{p}}_{k^{\prime}} \tilde{\boldsymbol{p}}_{k^{\prime}}^{T} \boldsymbol{c}_{k} \\
& +\sum_{l=1}^{L} \frac{N_{0}}{E_{l}^{(k)}} \frac{1}{\varsigma_{k l}}\left|\frac{h_{r l}^{(k)}}{h_{l}^{(k)}}\right|^{2} \boldsymbol{c}_{l}^{(k)}\left(\boldsymbol{c}_{l}^{(k)}\right)^{T}+\frac{N_{0}}{E_{r}^{(k)}} \boldsymbol{I}_{N} .
\end{aligned}
$$

Finally, when the signals received from both the first and second time slots of the $n$th symbol duration are combined, the decision variable for $b_{k}[n]$ can be formed as

$$
z_{k}[n]=w_{0}^{(k)} \bar{y}_{0}^{(k)}+\boldsymbol{w}^{H} \boldsymbol{y}_{1}^{(k)}, \quad k=1,2, \ldots, K
$$

where $\bar{y}_{0}^{(k)}$ and $w_{0}^{(k)}$ are given in (34) and (35).

\section{Power-Allocation}

As our study in [9] and [30] has shown, when large-scale fading is considered in the relay-assisted DS-CDMA systems, power should appropriately be allocated to the first and second time slots for the transmission of a symbol so that the DSCDMA systems are energy efficient and can achieve a nearbest error performance. To carry out a fair comparison between the relay-assisted DS-CDMA downlink and the conventional DS-CDMA downlink without using relays, as in [30], in this paper, we assume that the total transmission power is the same for the DS-CDMA systems either using or not using relays. Specifically, let $P_{0}=P_{k t}+L P_{l t}^{(k)}$ denote the total power radiated to MT $k$ by the DS-CDMA downlink without using relays, where $P_{k t}$ and $P_{l t}^{(k)}$ are detailed in the context of (5) and (20), respectively. Then, for the relay-aided DS-CDMA, with each MT helped by $L$ relays, $P_{k t}=\alpha P_{0}$ is allocated to the first time slot of a symbol for the BS to transmit signals to MT $k$ and its $L$ relays. During the second time slot of a symbol, the $L$ number of relays of MT $k$ uses the remaining power of $(1-\alpha) P_{0}$ to forward the signals received from the first time slot to MT $k$. Hence, the power allocated to each of the $L$ relays of MT $k$ is $P_{l t}^{(k)}=(1-\alpha) P_{0} / L$.

Note that, in our simulations in Section VII, we assume for simplicity that all the clusters of relays have a similar distance from the BS and that the relays within a cluster also have a similar distance from their common MT. We assume that, within each of the clusters, the large-scale statistics of the 


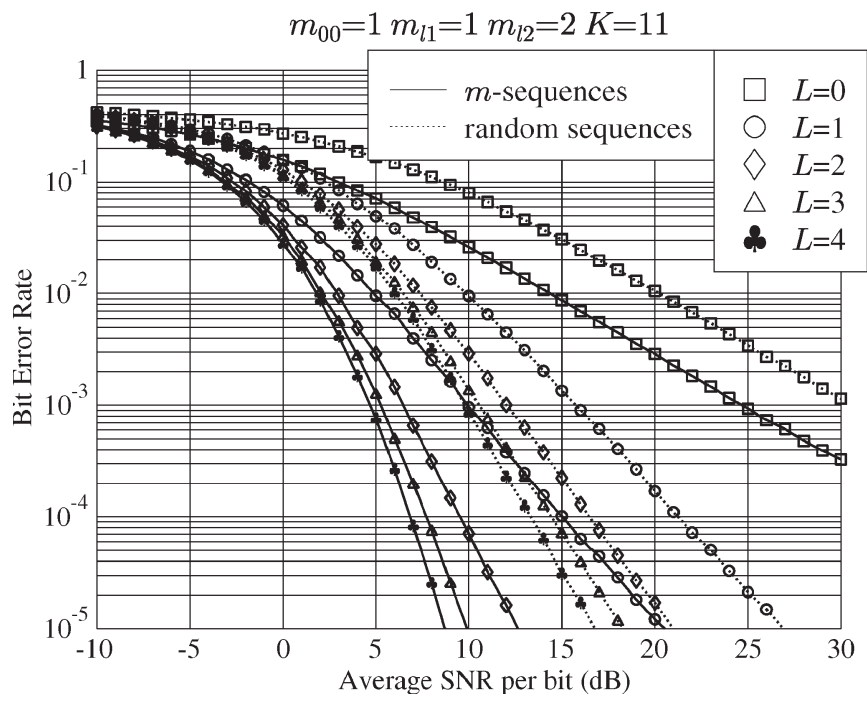

Fig. 2. BER versus SNR per bit performance of the relay-assisted DS-CDMA downlink using the TZF-based transmitter preprocessing at the BS and the MRC-SUC at MTs when the D-channels and BR-channels experience Rayleigh fading and the RM-channels experience Nakagami- $m$ fading associated with $m_{l 2}=2$ for $L=1,2,3$, and 4 . The other parameters used in our simulations were $N=15, K=11, \alpha=0.8, \delta=0.4$, and $\eta=3$.

received signals of an MT and its relays are similar. However, within a cluster, the signals transmitted by the relays to their MT are assumed to experience independent Nakagami- $m$ fading, implying that the small-scale statistics of the transmitted signals by the relays of a cluster are independent. In our simulations, we assume that all the distances concerned are normalized by the distance $d_{B M}$ between the BS and MT $k$. Specifically, we assume that the distance between the BS and MT $k$ is one unit, the distance between MT $k$ and its relays is $0<\delta<1$, and the distance between the relays of MT $k$ and the BS is $(1-\delta)$.

Note that the power-allocation scheme used in this contribution is the same as that proposed in [30] and [31], where the BER performance against the parameters $(\alpha, \delta)$ of the relays' location and power allocation for the relay-assisted DSCDMA system has been evaluated when communicating over the channels experiencing both propagation path loss and fast fading. It has been observed that the near-optimum values for the relays' location and power-allocation parameters are approximately $(\alpha=0.8, \delta=0.4)$ for the propagation path-loss exponent of $\eta=3$. Hence, in our simulations in Section VII, the parameters of $(\alpha=0.8, \delta=0.4)$ are adopted. Note that more details about the power allocation can be found in [30] and [31].

\section{Performance Results and Discussion}

In this section, we provide a range of simulation results to illustrate the BER performance of the relay-assisted DSCDMA downlink using the TZF- or TMMSE-based transmitter preprocessing. Figs. 2 and 3 depict the BER versus the average SNR per bit performance for the relay-assisted DS-CDMA downlink using the TZF-based transmitter preprocessing. The detection scheme employed by the destination MTs is the MRC-SUC in Fig. 2 and the MSINR-MUC in Fig. 3. The other parameters used in our simulations can be found in the captions of Figs. 2 and 3. From the results in Figs. 2 and 3, we may

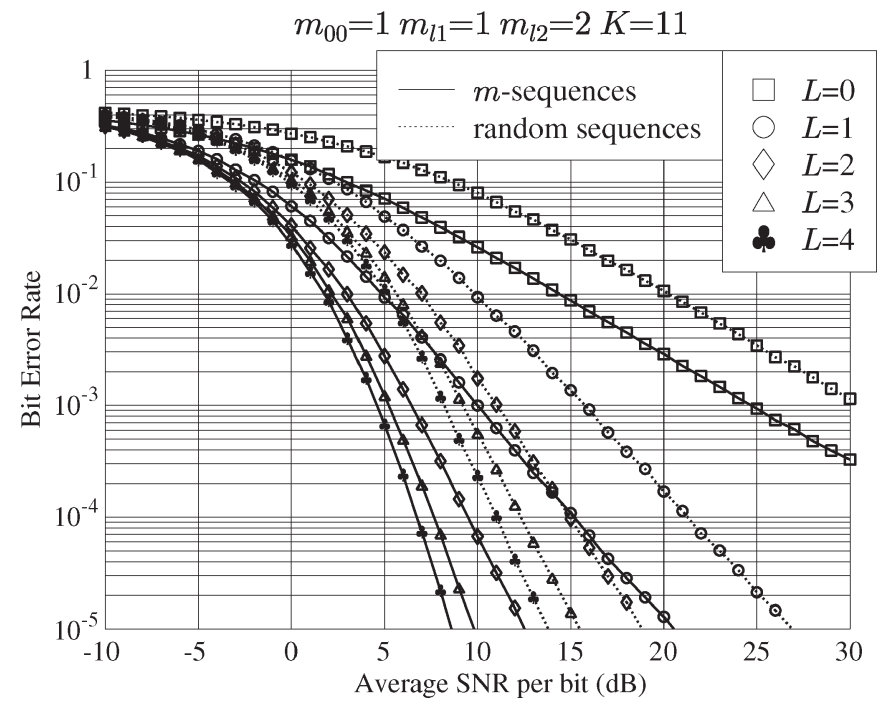

Fig. 3. BER versus SNR per bit performance of the relay-assisted DSCDMA downlink using the TZF-based transmitter preprocessing at the BS and the MSINR-MUC at MTs when the D-channels and BR-channels experience Rayleigh fading and the RM-channels experience Nakagami- $m$ fading associated with $m_{l 2}=2$ for $L=1,2,3$, and 4 . The other parameters used in our simulations were $N=15, K=11, \alpha=0.8, \delta=0.4$, and $\eta=3$.

obtain the following observations. First, the BER performance significantly improves when the number of relays per MT increases. Therefore, the relay-assisted DS-CDMA downlink is capable of achieving the relay diversity as promised. Second, for both random sequences and $m$-sequences, when $L=1$, the BER performance of using the MRC-SUC is the same as that of using the MSINR-MUC. This is because in this case, the MUI within the first time slot is fully removed by the TZFbased transmitter preprocessing, while there is no interference within the second time slot. Finally, when comparing Figs. 2 and 3, we can find that, when $m$-sequences are employed, the BER performance of the DS-CDMA downlink using both the MRC-SUC and the MSINR-MUC is similar when $L \geq 2$ relays per MT are employed. By contrast, when random sequences are utilized and when $L \geq 2$, the BER performance of the relayassisted DS-CDMA downlink using the MSINR-MUC is better than that of the relay-aided DS-CDMA downlink using the MRC-SUC.

In Figs. 4 and 5, we investigate the effect of the noisesuppression factor $\rho$ (where we assumed $\boldsymbol{\rho}=\rho \boldsymbol{I}_{K}$ ) on the achievable BER performance of the relay-assisted DS-CDMA downlink using the TMMSE-based transmitter preprocessing at the BS and the MRC-SUC at MTs when the D-channels and BR-channels experience Rayleigh fading and the RM-channels experience Nakagami- $m$ fading associated with $m_{l 2}=2$ for $L=1,2$, and 3 . The other parameters used in our simulations were $N=15, K=11, \alpha=0.8, \delta=0.4, \eta=3$, and SNR = $4 \mathrm{~dB}$ in Fig. 4 and $N=15, K=11, \alpha=0.8, \delta=0.4, \eta=3$, and SNR $=8 \mathrm{~dB}$ in Fig. 5. Note that, as shown in Section II, the TMMSE is reduced to the TZF when $\rho=0$. From the results in Figs. 4 and 5, we can observe that, for a given SNR, there exists an optimum $\rho$ value, which results in the lowest BER. However, the BER performance of the relay-assisted DSCDMA downlink using the TMMSE is not highly sensitive to the noise-suppression factor, particularly when $m$-sequences 


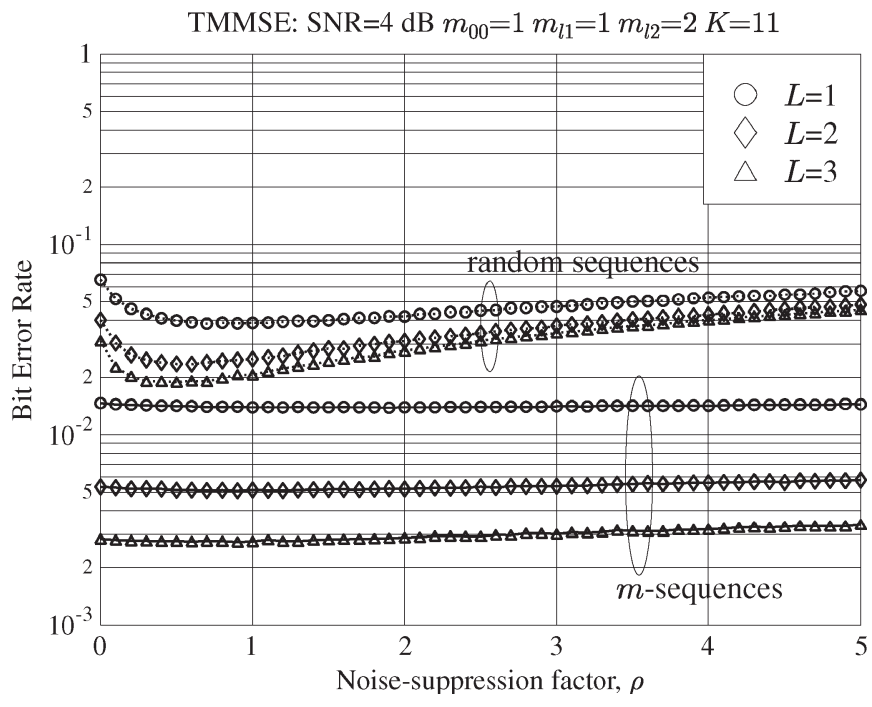

Fig. 4. BER versus the noise-suppression factor $\rho$ performance of the relayassisted DS-CDMA downlink using the TMMSE-based transmitter preprocessing at the BS and the MRC-SUC at MTs when the D-channels and BR-channels experience Rayleigh fading and the RM-channels experience Nakagami- $m$ fading associated with $m_{l 2}=2$ for $L=1,2$, and 3. The other parameters used in our simulations were $N=15, K=11, \alpha=0.8, \delta=0.4, \eta=3$, and $\mathrm{SNR}=4 \mathrm{~dB}$.

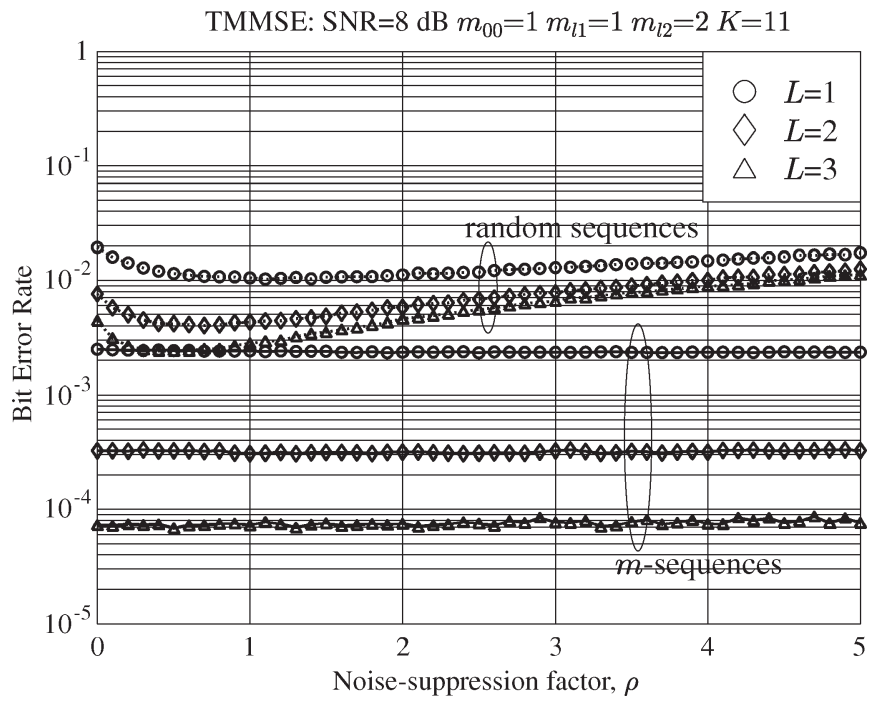

Fig. 5. BER versus the noise-suppression factor $\rho$ performance of the relayassisted DS-CDMA downlink using the TMMSE-based transmitter preprocessing at the BS and the MRC-SUC at MTs when the D-channels and BR-channels experience Rayleigh fading and the RM-channels experience Nakagami- $m$ fading associated with $m_{l 2}=2$ for $L=1,2$, and 3 . The other parameters used in our simulations were $N=15, K=11, \alpha=0.8, \delta=0.4, \eta=3$, and $\mathrm{SNR}=8 \mathrm{~dB}$

are used for spreading. As shown in Figs. 4 and 5, the BER is only loosely dependent on the $\rho$ value when random sequences are employed. Furthermore, from the results in Figs. 4 and 5 , we can observe that, for both $m$-sequences and random sequences, the BER becomes lower when an MT is aided by more relays. Hence, the diversity gain can be guaranteed for the relay-assisted DS-CDMA downlink if each destination MT can be aided by some relays.

Figs. 6 and 7 show the BER versus the average SNR per bit performance of the relay-assisted DS-CDMA downlink

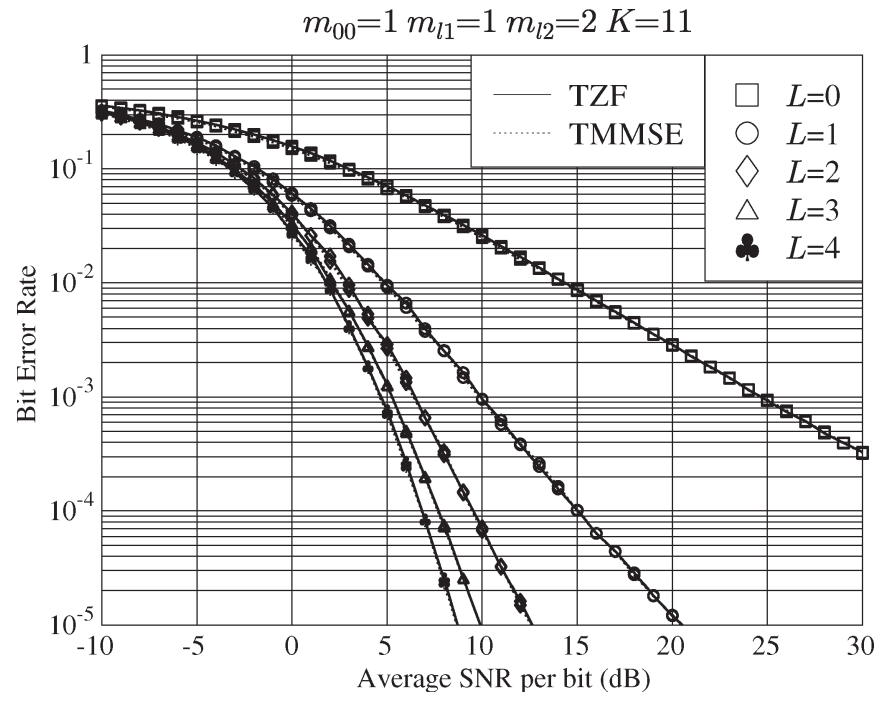

Fig. 6. BER versus SNR per bit performance of the relay-assisted DSCDMA downlink using $m$-sequences, the TZF- or TMMSE-based transmitter preprocessing at the BS, and the MRC-SUC at MTs when the D-channels and BR-channels experience Rayleigh fading and the RM-channels experience Nakagami- $m$ fading associated with $m_{l 2}=2$ for $L=1,2,3$, and 4 . The other parameters used in our simulations were $\rho=1.0$ for TMMSE, $N=15$, $K=11, \alpha=0.8, \delta=0.4$, and $\eta=3$.

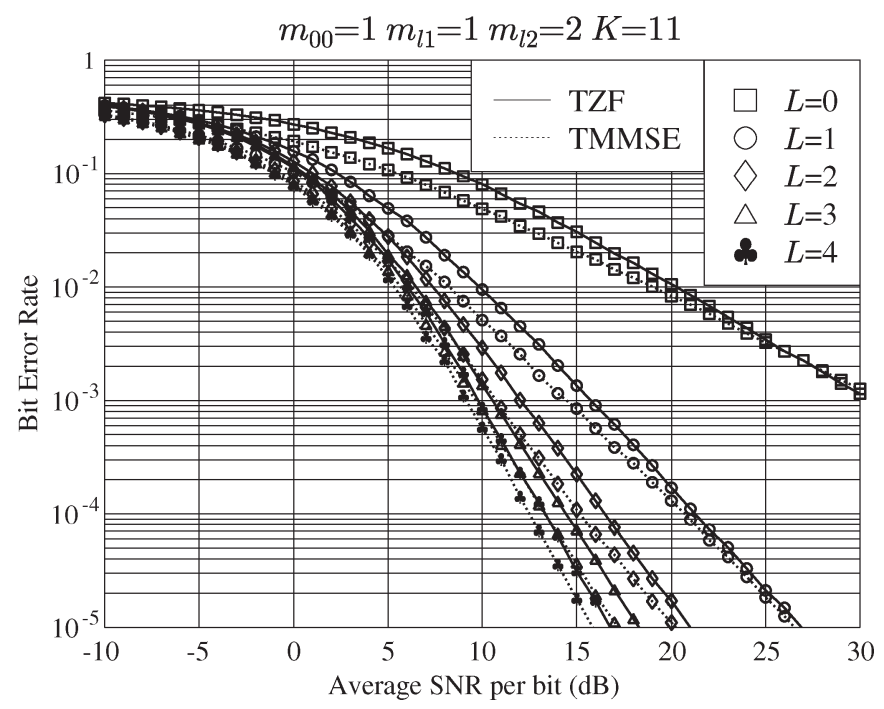

Fig. 7. BER versus SNR per bit performance of the relay-assisted DS-CDMA downlink using random sequences, the TZF- and TMMSE-based transmitter preprocessing at the BS, and the MRC-SUC at MTs when the D-channels and BR-channels experience Rayleigh fading and the RM-channels experience Nakagami- $m$ fading associated with $m_{l 2}=2$ for $L=1,2,3$, and 4 . The other parameters used in our simulations were $\rho=1.0$ for TMMSE, $N=15$, $K=11, \alpha=0.8, \delta=0.4$, and $\eta=3$.

using the TZF- or TMMSE-based transmitter preprocessing and the MRC-SUC detection. In our simulations in Fig. 6, $m$-sequences were assumed, while in Fig. 7, random sequences were employed. From the results in Figs. 6 and 7, it can be seen that the BER performance of the relay-assisted DS-CDMA downlink using both the TZF and the TMMSE is nearly the same when the $m$-sequences are employed for spreading. By contrast, when random spreading sequences are employed, the TMMSE outperforms the TZF. The reason for the aforementioned results may be stated as follows. We know that random 


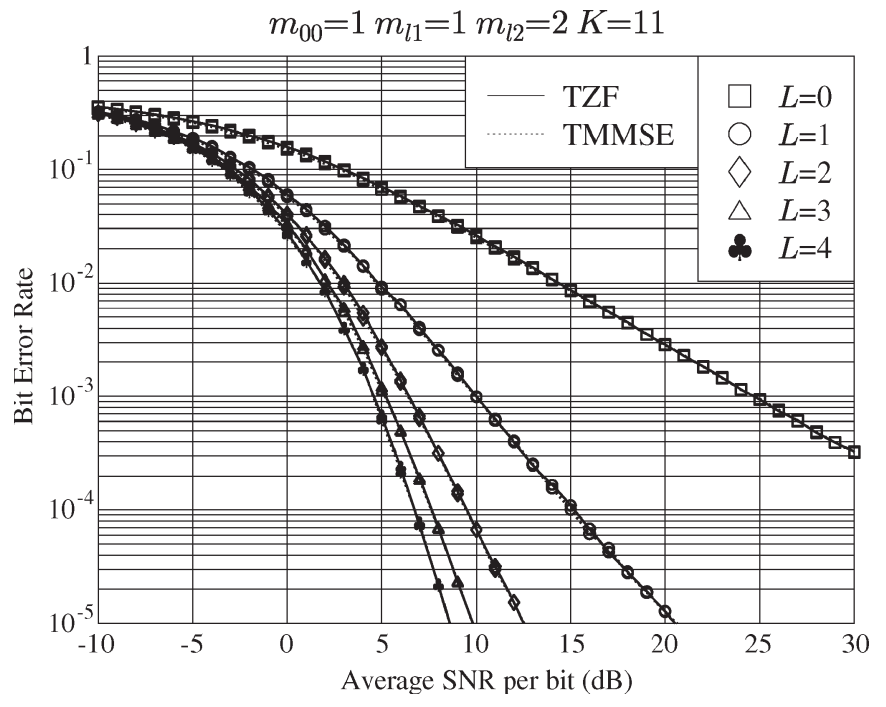

Fig. 8. BER versus SNR per bit performance of the relay-assisted DS-CDMA downlink using $m$-sequences, the TZF- and TMMSE-based transmitter preprocessing at the BS, and the MSINR-MUC at MTs when the D-channels and BR-channels experience Rayleigh fading and the RM-channels experience Nakagami- $m$ fading associated with $m_{l 2}=2$ for $L=1,2,3$, and 4 . The other parameters used in our simulations were $\rho=1.0$ for TMMSE, $N=15, K=11, \alpha=0.8, \delta=0.4$, and $\eta=3$.

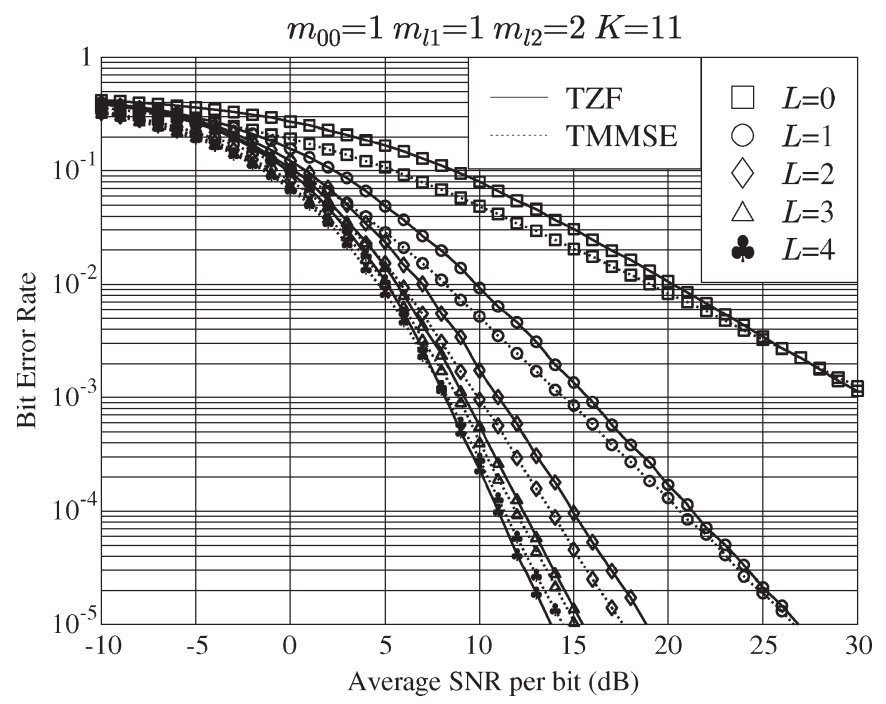

Fig. 9. BER versus SNR per bit performance of the relay-assisted DS-CDMA downlink using random sequences, the TZF- and TMMSE-based transmitter preprocessing at the BS, and the MSINR-MUC at MTs when the D-channels and BR-channels experience Rayleigh fading and the RM-channels experience Nakagami- $m$ fading associated with $m_{l 2}=2$ for $L=1,2,3$, and 4 . The other parameters used in our simulations were $\rho=1.0$ for TMMSE, $N=15$, $K=11, \alpha=0.8, \delta=0.4$, and $\eta=3$.

sequences generate higher MUI than $m$-sequences. It is well known that the TZF is capable of fully removing the downlink MUI but at the cost of noise amplification. By contrast, the TMMSE is capable of efficiently suppressing both the MUI and the background noise. Consequently, when random spreading sequences are employed, the TZF removes the MUI with severe noise amplification, resulting in BER performance degradation, in comparison with the TMMSE, which can suppress both the MUI and the background noise.

Finally, in Figs. 8 and 9, we evaluate the BER versus the average SNR per bit performance of the relay-assisted
DS-CDMA downlink using the TZF- or TMMSE-based transmitter preprocessing and the MSINR-MUC detection. The other parameters for the simulations in Figs. 8 and 9 are the same as those for the simulations in Figs. 6 and 7. From the results in Figs. 8 and 9, it can be observed that the BER performance of the relay-assisted DS-CDMA downlink using both the TZF and the TMMSE is nearly the same when $m$-sequences are used for spreading. This observation is similar to that seen in Fig. 6, where the MRC-SUC detection was employed. By contrast, when random spreading sequences are employed, the BER performance corresponding to the TMMSE is better than that corresponding to the TZF in low- to mediumSNR regions when $L \leq 3$. However, for $L=4$, the TMMSE is outperformed by the TZF when the SNR value is relatively high. The reason for the aforementioned observation is that, when random spreading sequences are employed, the leaked interference due to the TMMSE may become dominant, which cannot further be mitigated by the MSINR-MUC operated at the MTs. However, when comparing the results in Figs. 7 and 9, we can find that the MSINR-MUC always outperforms the MRC-SUC if two or more relays per MT are used.

\section{CONCLUSION}

In this paper, we have proposed and investigated a relayassisted DS-CDMA downlink transmission scheme, where the MUI is suppressed using the TZF- or TMMSE-based transmitter preprocessing. At the MTs, the signals received from the BS and relays are combined based on the maximal-ratio-combining or MSINR principles. From our analysis and simulation results, we can conclude that the relay diversity can be achieved after the MUI is mitigated using either the TZF- or TMMSEassisted transmitter preprocessing. It can be seen that when random spreading sequences are employed, the TMMSE-based transmitter preprocessing is capable of achieving better BER performance than the TZF-based transmitter preprocessing at low- to medium-SNR regions. By contrast, when $m$-sequences are employed, both transmitter preprocessing schemes achieve similar BER performance within the considered SNR region. Our simulation results show that, when the TMMSE-assisted transmitter preprocessing is employed, the BER performance is not very sensitive to the noise-suppression factor. Furthermore, in our proposed relay-diversity transmission scheme, the signal processing required by the relays is of low complexity.

\section{ACKNOWLEDGMENT}

The authors would like to thank the anonymous reviewers for their helpful comments and valuable suggestions.

\section{REFERENCES}

[1] S. M. Alamouti, "A simple transmit diversity technique for wireless communications," IEEE J. Sel. Areas Commun., vol. 16, no. 8, pp. 1451-1458, Oct. 1998.

[2] V. Tarokh, N. Seshadri, and A. R. Calderbank, "Space-time codes for high data rate wireless communication: Performance criterion and code construction," IEEE Trans. Inf. Theory, vol. 44, no. 2, pp. 744-765, Mar. 1998. 
[3] V. Tarokh, H. Jafarkhani, and A. R. Calderbank, "Space-time block coding for wireless communications: Performance results," IEEE J. Sel. Areas Commun., vol. 17, no. 3, pp. 451-460, Mar. 1999.

[4] L.-L. Yang, Multicarrier Communications. Chichester, U.K.: Wiley, 2009.

[5] R. U. Nabar and H. Bölcskei, "Space-time signal design for fading relay channels," in Proc. IEEE GLOBECOM, San Francisco, CA, Dec. 2003, vol. 4, pp. 1952-1956.

[6] I. E. Telatar, "Capacity of multiantenna Gaussian channels," Eur. Trans. Telecommun., vol. 10, no. 6, pp. 585-595, Nov./Dec. 1999.

[7] B. Hochwald, T. L. Marzetta, and C. B. Papadias, "A transmitter diversity scheme for wideband CDMA systems based on space-time spreading," IEEE J. Sel. Areas Commun., vol. 19, no. 1, pp. 48-60, Jan. 2001.

[8] L.-L. Yang and L. Hanzo, "Performance of broadband multicarrier DS-CDMA using space-time spreading-assisted transmit diversity," IEEE Trans. Wireless Commun., vol. 4, no. 3, pp. 885-894, May 2005.

[9] W. Fang, L. L. Yang, and L. Hanzo, "Single-user performance of directsequence code-division multiple-access using relay diversity and power allocation," IET Commun., vol. 2, no. 3, pp. 462-472, Mar. 2008.

[10] A. Sendonaris, E. Erkip, and B. Aazhang, "Increasing uplink capacity via user cooperation diversity," in Proc. IEEE Int. Symp. Inf. Theory, Cambridge, MA, Aug. 1998, p. 156.

[11] A. Sendonaris, E. Erkip, and B. Aazhang, "User cooperation diversity-Part I: System description," IEEE Trans. Commun., vol. 51, no. 11, pp. 1927-1938, Nov. 2003.

[12] A. Sendonaris, E. Erkip, and B. Aazhang, "User cooperation diversity-Part II: Implementation aspects and performance analysis," IEEE Trans. Commun., vol. 51, no. 11, pp. 1939-1948, Nov. 2003.

[13] P. A. Anghel and M. Kaveh, "Exact symbol error probability of a cooperative network in a Rayleigh-fading environment," IEEE Trans. Wireless Commun., vol. 3, no. 5, pp. 1416-1421, Sep. 2004.

[14] M. O. Hasna and M. S. Alouini, "Harmonic mean and end-to-end performance of transmission systems with relays," IEEE Trans. Commun., vol. 52, no. 1, pp. 130-135, Jan. 2004.

[15] B. R. Vojcic and W. M. Jang, "Transmitter precoding in synchronous multiuser communications," IEEE Trans. Commun., vol. 46, no. 10, pp. 1346-1355, Oct. 1998.

[16] W. Fang, L.-L. Yang, and L. Hanzo, "Performance of DS-CDMA downlink using transmitter preprocessing and relay diversity over Nakagami-m fading channels," IEEE Trans. Wireless Commun., vol. 8, no. 2, pp. 678682, Feb. 2009.

[17] L.-U. Choi and R. D. Murch, "Transmit-preprocessing techniques with simplified receivers for the downlink of MISO TDD-CDMA systems," IEEE Trans. Veh. Technol., vol. 53, no. 2, pp. 285-295, Mar. 2004.

[18] L. Li and G. Gu, "Design of optimal zero-forcing precoders for MIMO channels via optimal full information control," IEEE Trans. Signal Process., vol. 53, no. 8, pp. 3238-3246, Aug. 2005.

[19] L.-L. Yang, "A zero-forcing multiuser transmitter preprocessing scheme for downlink communications," IEEE Trans. Commun., vol. 56, no. 6, pp. 862-865, Jun. 2008.
[20] A. Klein, G. K. Kaleh, and P. W. Baier, "Zero forcing and minimum mean-square-error equalization for multiuser detection in code-division multiple-access channels," IEEE Trans. Veh. Technol., vol. 45, no. 2, pp. 276-287, May 1996.

[21] S. Sud, W. Myrick, P. Cifuentes, J. Goldstein, and M. Zoltowski, "A low complexity MMSE multiuser detector for DS-CDMA," in Conf. Rec. 35th Asilomar Conf. Signals, Syst. Comput., Pacific Grove, CA, Nov. 2001, vol. 1, pp. 404-409.

[22] L.-L. Yang, "Design linear multiuser transmitters from linear multiuser receivers," in Proc. IEEE ICC, Glasgow, U.K., Jun. 2007, pp. 5258-5263.

[23] J. G. Proakis, Digital Communications, 4th ed. New York: McGraw-Hill, 2000.

[24] S. Verdù, Multiuser Detection. Cambridge, U.K.: Cambridge Univ. Press, 1998.

[25] H. L. V. Trees, Optimum Array Processing. New York: WileyInterscience, 2002.

[26] T. S. Rappaport, Wireless Communications: Principles and Practice. Upper Saddle River, NJ: Prentice-Hall, 1996.

[27] D. Reynolds, X. Wang, and K. N. Modi, "Interference suppression and diversity exploitation for multiantenna CDMA with ultra-low complexity receivers," IEEE Trans. Signal Process., vol. 53, no. 8, pp. 3226-3237, Aug. 2005.

[28] N. Nakagami, "The $m$-distribution, a general formula for intensity distribution of rapid fading," in Statistical Methods in Radio Wave Propagation, W. G. Hoffman, Ed. Oxford, U.K.: Pergamon, 1960.

[29] X. Wang and H. V. Poor, Wireless Communication Systems-Advanced Techniques for Signal Reception. Englewood Cliffs, NJ: Prentice-Hall, 2003.

[30] W. Fang, L.-L. Yang, and L. Hanzo, "Single-user performance of relayassisted DS-CDMA with power allocation and inter-relay interference suppression," in Proc. IEEE VTC_Fall, Baltimore, MD, Sep./Oct. 2007, pp. 1004-1008.

[31] W. Fang, L.-L. Yang, and L. Hanzo, "Performance of relay-aided DS-CDMA experiencing propagation pathloss and Nakagami fading," in Proc. IEEE VTC-Fall, Calgary, AB, Canada, Sep. 2008, pp. 1-5.

Wei Fang (A'08), photograph and biography not available at the time of publication.

Lie-Liang Yang (M'98-SM'02), photograph and biography not available at the time of publication.

Lajos Hanzo (M'91-SM'92-F'04), photograph and biography not available at the time of publication. 